Proceedings of the International School and Conference on Optics and Optical Materials, ISCOM07, Belgrade, Serbia, September 3-7, 2007

\title{
Dispersion of Bloch Modes in a Multilayer Structure with Alternating Left-Handed and Right-Handed Materials
}

\author{
S. Vukovic*, N.B. Aleksic and D.V. Timotijevic \\ Institute of Physics, Pregrevica 118, 11080 Belgrade, Serbia
}

\begin{abstract}
We study all-evanescent eigenmodes of a one-dimensional infinite periodic structure with alternating left-handed and right-handed materials that propagate perpendicularly to the surface or guided eigenmodes. Investigation of dispersion properties of such Bloch modes is shown to be crucial for understanding of an efficient radiation energy transport across the periodic multilayer structure. Frequency pass bands and gaps are found as a function of the two orthogonal wave vectors: the Bloch wave vector $\boldsymbol{k}_{\mathrm{B}}$ and the surface wave vector $\boldsymbol{k}_{\mathrm{S}}$. We demonstrate that pass bands of both TE- and TM-polarizations can exist and, under certain conditions, may overlap.
\end{abstract}

PACS numbers: 41.20. Jb, 78.66. Fd, 78.68.+m

\section{Introduction}

In an isotropic medium with a negative dielectric permittivity $\varepsilon(\omega)<0$ and negative magnetic permeability $\mu(\omega)<0$ over a common band of frequencies $\omega$, an electromagnetic wave propagates with the wave vector $\boldsymbol{k}$, the electric field $\boldsymbol{E}$, and the magnetic field $\boldsymbol{H}$ that form a left-handed orthogonal set. Such a medium is labeled as left-handed ( $\mathrm{LH})$, as opposite to the conventional right-handed ( $\mathrm{RH})$ media. In addition, there appears a negative group velocity (directed oppositely to the wave phase velocity) as an intrinsic property of electromagnetic wave propagation in LH media. In fact, the term "left-handed media" was introduced by Veselago [1] to illustrate that propagation of light, under such conditions, can exhibit very peculiar properties: negative refraction, reversed Doppler shift and inverse Cherenkov radiation. The concept of a negative refractive index was introduced by the same author [2], too. However, the concept of negative group velocity of an electromagnetic radiation was introduced by Mandel'shtam [3], much earlier. No naturally existing LH medium has yet been discovered. Therefore, it became

*corresponding author; e-mail: svukovic@phy.bg.ac.yu 
necessary to create artificial, man made materials, called metamaterials [4]. It is worth noting that actual LH media are composed of unit parts of finite dimensions and thus are heterogeneous. In order to consider them as continuous media and introduce an effective dielectric permittivity and effective magnetic permeability that are simultaneously negative, it becomes necessary to observe the homogenization limit [5]. Nevertheless, the optical properties of LH media have attracted considerable attention in the past several years in efforts to develop novel optical devices, including perfect lenses [6].

In the present paper we consider eigenmodes of a one-dimensional multilayer periodic structure with alternating LH metamaterial and conventional dielectric layers, with the wave vector $\boldsymbol{k}_{\mathrm{B}}$ that is oriented normally to the surface between alternating media. At the same time, there are eigenmodes that propagate parallel to the surface (with the wave vector $\boldsymbol{k}_{\mathrm{S}}$ ) in the form of surface or guided waves with the field amplitude that is evanescent away from the surface. In other words, we investigate the Bloch modes with all-evanescent fields intrinsically coupled with the surface or guided modes that propagate perpendicularly. Diagrams of photonic bands and gaps describe the properties of an infinite periodic structure [7]. Unlike conventional pass bands in photonic crystals, where the finite thickness of the structure affects the transmission of light having frequency within the pass band [8], the properties of the transparent band that can be designed into a 1D metallodielectric nanofilm structure $[9,10]$ remain almost unaffected by the finite thickness of the structure. In contrast to metallodielectric multilayer structure, where the transparency band corresponds to the TM polarization only, we demonstrate that alternating LH-RH structure reveal frequency bands for both TE- and TM-polarizations that may overlap under certain conditions.

\section{Formulation of the problem}

We consider a 1D infinite periodic structure with alternating layers of conventional dielectric (subscript 1) and left-handed material (subscript 2) as presented in Fig. 1. The relative dielectric permittivity $\varepsilon_{1}$ and permeability $\mu_{1}=1$ are considered to be non-dispersive, while $\varepsilon_{2}$ and $\mu_{2}$ have to be frequency dependent and simultaneously negative over a common band of frequencies. In the present paper, neglecting dissipation, we will adopt the following expressions [4]:

$$
\varepsilon_{2}=1-\bar{\omega}^{-2}, \quad \mu_{2}=1-F \bar{\omega}^{2} /\left(\bar{\omega}^{2}-G\right) .
$$

Here, $\bar{\omega}^{2}=\omega^{2} / \omega_{\mathrm{pe}}^{2}, \omega_{\mathrm{pe}}^{2}$ being the electron plasma frequency, $F=0.56$ and $G=0.16$. The thicknesses of the dielectric and left-handed layers are denoted by $a$ and $d$, respectively, with a period $a+d+a=2 a+d$, being the thickness of the unit cell of the multilayer structure. If the number of periods is infinite, the configuration of unit cell is not so important. However, the termination of the multilayer structure becomes very important for finite number of periods in terms of the transparent photonic band. In the present paper we confine ourselves to infinite number of periods, but keep the configuration of the unit cell suitable for 

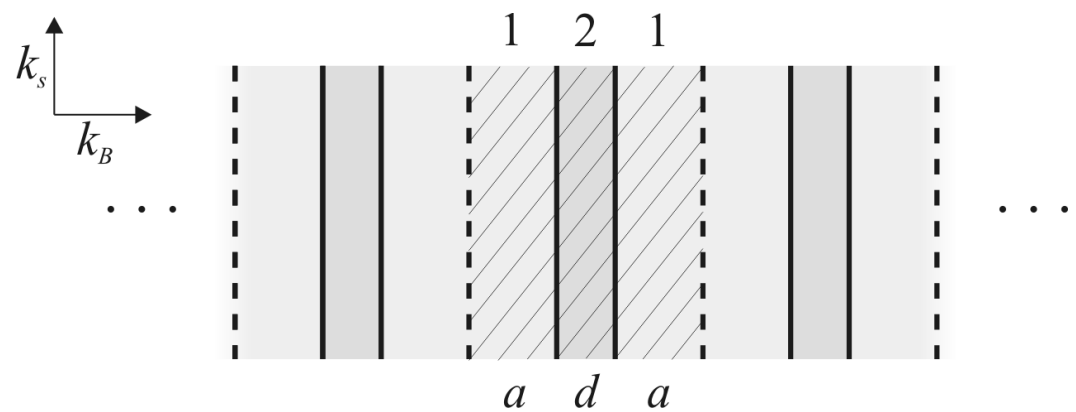

Fig. 1. Geometry of the problem. Shaded area represents the unit cell of the multilayer structure.

the investigation of the transparent photonic bands and gaps. In order to study dispersion of the Bloch modes it is suitable to introduce non-dimensional wave numbers $\bar{k}_{\mathrm{S}}=k_{\mathrm{S}} c / \omega_{\text {pe }}$ and $\bar{k}_{\mathrm{B}}=k_{\mathrm{B}} c / \omega_{\text {pe }}$, as well as layer thicknesses $\bar{a}=\omega_{\mathrm{pe}} a / c$ and $\bar{d}=\omega_{\text {pe }} d / c, c$ being the speed of light in vacuum. It is our aim to find $\bar{\omega}$ as a function of $\bar{k}_{\mathrm{S}}$ and $\bar{k}_{\mathrm{B}}$ in the frequency region $0.4<\bar{\omega}<0.6$, where both $\varepsilon_{2}$ and $\mu_{2}$ are simultaneously negative. At the same time, we investigate all-evanescent fields that are intrinsically coupled with surface or guided modes which means $\bar{k}_{\mathrm{S}}>\left(\varepsilon_{1} \mu_{1}\right)^{1 / 2} \bar{\omega} ; \bar{k}_{\mathrm{S}}>\left[\varepsilon_{2}(\bar{\omega}) \mu_{2}(\bar{\omega})\right]^{1 / 2} \bar{\omega}$. The existence of the Bloch modes and the corresponding transparency bands for the TE-, as well as for TM-polarization, opens up the possibility of total transmission of light through the classically opaque multilayer media.

\section{Dispersion of the Bloch modes}

According to the general theory that has been presented in Ref. [7], one can find the following dispersion relation for the TE-polarized Bloch mode:

$$
\cos \Omega=\cosh \left(2 \kappa_{1} \bar{a}\right) \cosh \left(\kappa_{2} \bar{d}\right)+A \sinh \left(2 \kappa_{1} \bar{a}\right) \sin \left(\kappa_{2} \bar{d}\right),
$$

where $\Omega=\bar{k}_{\mathrm{B}}(2 \bar{a}+\bar{d}), A=\frac{\left(\kappa_{1} \mu_{2}\right)^{2}+\left(\kappa_{2} \mu_{1}\right)^{2}}{2 \kappa_{1} \mu_{1} \kappa_{2} \mu_{2}}, \kappa_{1,2}=\sqrt{\bar{k}_{\mathrm{S}}^{2}-\bar{\omega}^{2} \varepsilon_{1,2} \mu_{1,2}}$. The corresponding equation for the TM-polarization can be obtained via mutual replacement $\mu_{1,2} \rightleftharpoons \varepsilon_{1,2}$. For real values of the Bloch wave number $k_{\mathrm{B}}$, the right hand side of Eq. (2) can take the values between -1 and 1 . In that case, $k_{\mathrm{B}}$ changes in the range $0<\bar{k}_{\mathrm{B}}<\pi /(2 \bar{a}+\bar{d})$. Contour plots of $\bar{\omega}$ as a function of $\bar{k}_{\mathrm{S}}$ for various $\bar{k}_{\mathrm{B}}$ are presented in Fig. $2 \mathrm{a}-\mathrm{f}$. For each of the two orthogonal polarizations, the results depend on the three parameters, $\varepsilon_{1}, \bar{a}$, and $\bar{d}$, because $\mu_{1}=1$ has been assumed everywhere. In Fig. 2, however, we fix $\bar{a}=1.2$ and $\bar{d}=0.8$, but change $\varepsilon_{1}$ from 2 in Fig. $2 \mathrm{a}$ and b to 5 in Fig. 2e and f. In general, there are two branches of the contour plots: the upper branch and the lower one. As can be seen, when $\bar{k}_{\mathrm{S}}$ increases those two branches become more and more narrow, and finally, both tend to a single solid line(s) labeled 3 in Fig. 2, that represents the dispersion curve of the surface waves $(a, d \rightarrow \infty)$. Therefore, it is more interesting to study the lower values of $\bar{k}_{\mathrm{S}}$ for the frequency pass bands. Also, from the point 
of view of the potential applications, the lower values of $\bar{k}_{\mathrm{S}}$ are much easier to excite, e.g. via attenuated total reflection (ATR) technique. Let us notice that the group velocity of the surface wave changes its sign when $\varepsilon_{1}$ passes through the critical value $\varepsilon_{1}=3.5$, when the group velocity of the surface wave becomes equal to zero. Nevertheless, since $\bar{a}$ and $\bar{d}$ are finite, the upper and lower branches exist even for the critical value of $\varepsilon_{1}$. In fact, they correspond to the fundamental symmetric and first antisymmetric slow guided modes in left-handed waveguides, respectively [11].

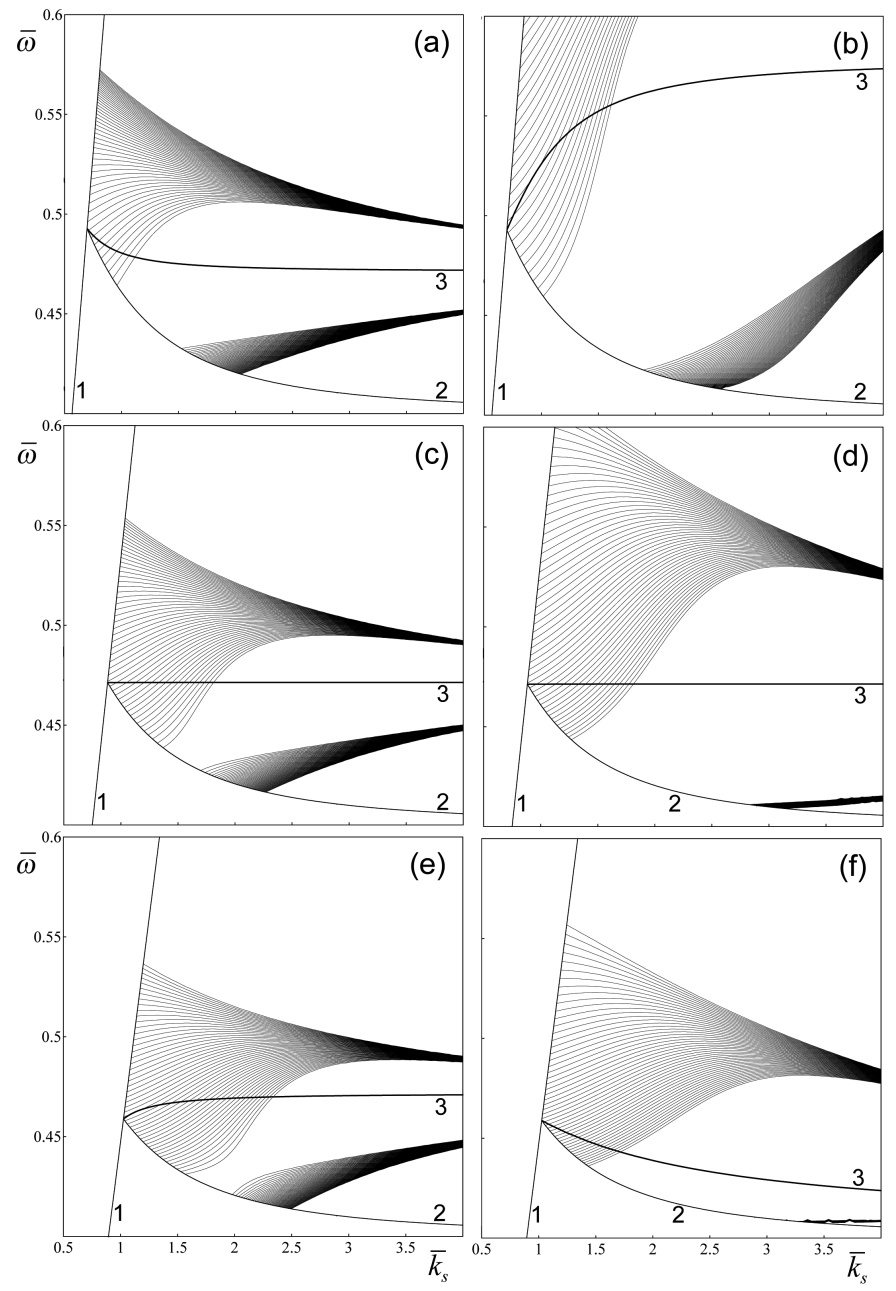

Fig. 2. Contour plots of the frequency $\bar{\omega}$ versus surface wave number $\bar{k}_{\mathrm{S}}$ for given real values of the Bloch wave number $\bar{k}_{\mathrm{B}}$. TE-polarization: (a), (c), and (e); TM-polarization: (b), (d), and (f). In all figures $\bar{a}=1.2, d=0.8 . \quad \varepsilon_{1}=2$ in (a) and (b); $\varepsilon_{1}=3.5$ in (c) and (d); $\varepsilon_{1}=5$ in (e) and (f). The lines labeled 1 and 2 represent dispersion curves of the bulk waves in medium 1 and 2 , respectively. The lines labeled 3 are dispersion curves of the surface waves. 
However, the most striking feature of Fig. 2 is the existence of the overlapping parts of the TE- and TM-polarized pass bands.

To complete the picture of the dispersion of the Bloch modes, we present in Fig. 3 the frequency $\bar{\omega}$ as a function of the Bloch wave number $\bar{k}_{\mathrm{B}}$ for the fixed value of $\bar{k}_{\mathrm{S}}=2$. The upper and the lower branches of the transparent frequency bands are clearly seen for both TE- and TM-polarizations. However, the existence of the frequency gaps can be seen much more clearly than in Fig. 2.

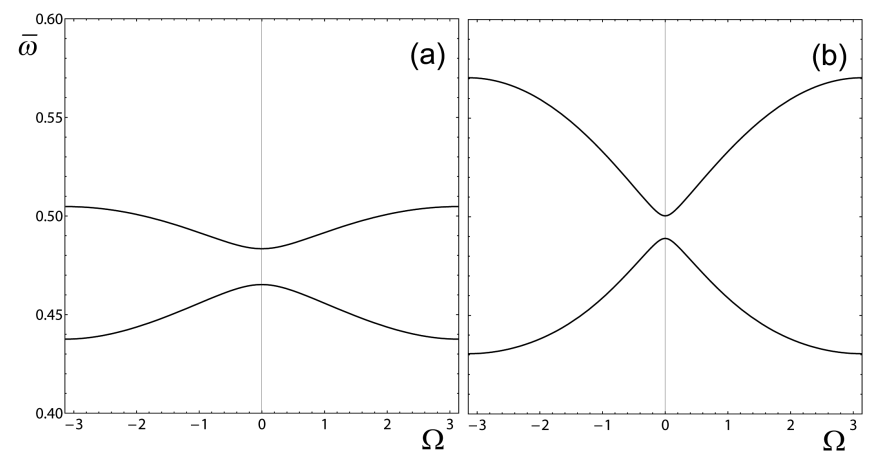

Fig. 3. Dispersion of the Bloch waves; frequency $\bar{\omega}$ as a function of the Bloch wave number $\bar{k}_{\mathrm{B}}$. Parts (a) and (b) correspond to TE- and TM-polarization, respectively. In both (a) and (b) $\bar{a}=1.2, \bar{d}=1.8, \varepsilon_{1}=2.5$, and $\bar{k}_{\mathrm{S}}=2$.

When $\bar{k}_{\mathrm{S}}$ decreases, the lower branch can disappear, but the upper branch remains. Here, the parameters $\bar{d}$ and $\varepsilon_{1}$ have been chosen to be different from those presented in Fig. 2. Nevertheless, the general behavior looks the same in both Figs. 2 and 3. This is not surprising since Figs. 2 and 3 represent just different views on the same physical picture.

\section{Conclusion}

Dispersion properties of the Bloch modes with all-evanescent fields that can propagate perpendicularly to the surface or guided modes in a one-dimensional infinite periodic multilayer structure with alternating left-handed and right-handed materials have been investigated. Transparent frequency bands, as well as frequency gaps have been found for both TE- and TM-polarizations and there are always two branches: the upper one and the lower one, with respect to the surface wave dispersion curve. It has been demonstrated that the frequency bands of the two orthogonal polarizations can overlap. In that case, the resonant excitation of the Bloch modes with all-evanescent fields in a structure that consists of a finite number of unit cells is expected to reveal total transparency of the classically opaque layered media. 


\section{Acknowledgments}

The authors acknowledge a financial support of the Ministry of Science of the Republic of Serbia (grant No. OI 141031).

\section{References}

[1] V.G. Veselago, Usp. Fiz. Nauk 92, 517 (1967) [Sov. Phys. Usp. 10, 509 (1968)].

[2] V.G. Veselago, Fiz. Tverd. Tela 8, 3571 (1966) [Sov. Phys.-Solid State 8, 2853 (1967)].

[3] L.I. Mandel'shtam, Zh. Eksp. Teor. Fiz. 15, 475 (1945).

[4] D.R. Smith, W.J. Padilla, S.C. Vier, S.C. Nemat-Naser, S. Schultz, Phys. Rev. Lett. 84, 4184 (2000).

[5] D. Maystre, S. Enoch, J. Opt. Soc. Am. A 21, 122 (2004).

[6] J.B. Pendry, Phys. Rev. Lett. 85, 3966 (2000).

[7] J.M. Bendickson, J.P. Dowling, M. Scalora, Phys. Rev. E 53, 4107 (1996).

[8] J.M. Elson, K. Halterman, Opt. Express 12, 4885 (2004).

[9] S. Feng, J.M. Elson, P.L. Overfelt, Opt. Express 13, 4113 (2005).

[10] S. Feng, J.M. Elson, P.L. Overfelt, Phys. Rev. B 72, 085117 (2005).

[11] I.V. Shadrivov, A.A. Sukhorukov, Yu.S. Kivshar, Phys. Rev. E 67, 057602 (2003). 